\title{
The power and limitations of genomic surveillance of bacteria
}

\author{
Fernando González-Candelas* ${ }^{* 1,2,3}$ (D) Carlos Francés-Cuesta ${ }^{1,2}$ (i) \& Neris \\ García-González ${ }^{1,2}$ (D) \\ ${ }^{1}$ Institute for Integrative Systems Biology, I2SysBio (CSIC-UV), University of Valencia, Valencia, Spain \\ ${ }^{2}$ Joint Research Unit 'Infection \& Public Health' FISABIO-University of Valencia, Valencia, Spain \\ ${ }^{3}$ CIBER in Epidemiology \& Public Health, Valencia, Spain \\ *Author for correspondence: fernando.gonzalez@uv.es
}

“The progressive adoption of WGS as a regular, even routine, technique in clinical microbiology laboratories and the deposit of those sequences along with basic information in public databases will greatly facilitate epidemiological surveillance"

First draft submitted: 28 September 2019; Accepted for publication: 28 November 2019; Published online: 19 December 2019

Keywords: antimicrobial resistance $\bullet$ carbapenemases $\bullet$ genomic surveillance $\bullet$ Klebsiella pneumoniae $\bullet$ microbial genome $\bullet$ molecular epidemiology $\bullet$ Neisseria gonorrhoeae $\bullet$ next-generation sequencing

\section{Why genomic surveillance?}

Genomic surveillance is becoming the new gold standard for the analysis, vigilance and control of pathogens. The decreasing costs, faster turnaround times, increased accuracy and availability of easy-to-use tools [1] for the results obtained by high-throughput sequencing of genomes have led to an impressive accumulation of whole genome sequences (WGS) in the past 5 years. This has facilitated the use of WGS data as a rich source of information for epidemiological surveillance of infectious pathogens, most notably some viruses - such as influenza A [2], hepatitis C [3] or human immunodeficiency viruses [4] - and bacteria. In fact, the European Center for Disease Prevention and Control recently announced that it will be progressively adopting WGS as the method of choice for surveillance of antimicrobial-resistant pathogens.

Two recent publications have reported large scale, retrospective analyses of bacterial pathogens, carbapenemaseproducing Klebsiella pneumoniae [5] and Neisseria gonorrhoeae [6], respectively. They belong to two of the most relevant groups of pathogens from a public health perspective, antimicrobial-resistant (AMR) and sexually transmitted infectious (STI) bacteria. AMR poses a major threat, not only for public health at a global scale, but also with direct economic and social consequences. Carbapenamase-producing Enterobacteriaceae are among the most worrisome groups of resistant bacteria, not only because they have less sensitivity to second- and third-line antibiotics, but also because they tend to accumulate resistance factors, leading to extreme drug resistance that drastically limits the possible therapeutic options. STIs are on the rise all over the world, with a steep increase in the last decade. This is largely due to an expanding epidemic in several high-risk groups, globally defined by a high frequency of unprotected sexual contacts. Occasionally, both factors concur in the same pathogen, potentially leading to a 'perfect storm' scenario, with a fast expansion in sexual networks of an AMR pathogen. This is the case of some strains of $N$. gonorrhoeae $[7,8]$. The increase of resistance and the limitations of therapeutic options for these pathogens are the reason for including $K$. pneumoniae and $N$. gonorrhoeae in the WHO priority list for research and development of new antibiotics and also why it is important to invest in their surveillance and control.

\section{Genomic surveillance of $K$. pneumoniae}

The papers by David et al. [5] and Sánchez-Busó et al. [6] are excellent examples of the benefits of WGS for surveillance and of how the same basic data can provide information at different levels. They share some features, such as large sample sizes, wide geographic and temporal distribution of isolates and rigorous methods of analysis, that lead to well-supported but unanticipated conclusions. For instance, David et al. reveal the central role of intra-

Future $\because \cdots$ Medicine 
and interhospital transmissions for the expansion of the most burdensome lineages of $K$. pneumoniae. This result is at odds with previous ideas according to which animals and environmental reservoirs likely played a major role in the dissemination of these clones in human populations [9]. The 'One Health' perspective for surveillance does not seem to be the best strategy in the case of $K$. pneumoniae, at least for these high-risk lineages.

Two additional conclusions from this study are also conflicting with practical applications of genomic surveillance. One is the relatively large number of discrepancies between the results of phenotypic and genotypic tests for antibiotic resistance, with over $20 \%$ of cases of nonsusceptible strains reported by hospitals lacking any known gene explaining this phenotype. Apart from the possible explanations discussed by the authors, this figure may lead to questioning the validity of the current methods, both phenotypic and genome-based, in their application in the clinical setting. The other relevant conclusion is the definition of an optimal threshold, in this case 21 single-nucleotide polymorphisms, for identifying potential hospital clusters. Should this or any other value of genetic distance identify nosocomial outbreaks correctly, the genomic surveillance task would be greatly facilitated. However, and this reminds us one important limitation of genomic surveillance, a lack of epidemiological data prevented the authors from contrasting the validity and generality of their conclusion with actual information about contacts and transmission networks.

\section{Genomic surveillance of $\boldsymbol{N}$. gonorrhoeae}

Sánchez-Busó et al. analyzed a smaller sample $(419 \mathrm{~N}$. gonorrhoeae strains compared with $1717 \mathrm{~K}$. pneumoniae isolates) but with a wider geographic (58 countries of five continents compared with 32 countries in Europe plus Turkey and Israel) and temporal (50 years compared with 6 months in the $K$. pneumoniae study) range than David et al. The sampling designs in these studies were also different. In Sánchez-Busó et al., the main objective was to maximize the diversity of the studied isolates, in order to draw conclusions about the global spread and evolution of $N$. gonorrhoeae. Instead, David et al. aimed at characterizing an instant snapshot of the genomic diversity of K. pneumoniae in the context of European Committee on Antimicrobial Susceptibility Testing (EUCAST). These different goals are also reflected in the scope of the conclusions derived from both studies. For instance, SánchezBusó et al. infer that the most recent common ancestor of the analyzed strains can be dated to the 16th century. This result questions the validity of prevailing ideas about an ancient origin of gonorrhea, 'as old as humankind'. The beginning of the Modern Era marked a time of change at many levels, with a dramatic increase in transoceanic trips. This led to an exchange of pathogens between previously isolated continents which resulted in epidemic expansions and the 'emergence' of new infections. The currently circulating strains of two of these, Treponema pallidum subsp. pallidum, the causative agent of another STI, syphilis, and $N$. gonorrhoeae, originated in this period $[6,10]$. This does not mean that this was the time of the first infections in humans by these pathogens, but that the currently circulating strains, or at least those whose genome sequence has been determined so far, derive from one strain circulating at that time, their 'mother Eve'. Whether the replacement of previous lineages was complete or the role of epidemic and evolutionary processes (selection of more virulent or transmissible variants, chance events in the early stages of expansion, incorporation to epidemiologically successful transmission routes, etc.) will be elucidated only through the analysis of many more isolates and the recovery of genome sequences from ancient remains.

The connections between humans and pathogens has left evolutionary footprints in both sides. Apart from the obvious results of increasing selective pressure arising from antibiotics, bacteria can also respond to other human-mediated changes. For instance, Sánchez-Busó et al. differentiate two major lineages of N. gonorrhoeae, denoted A and B. Apart from the genetic variants that separate them, they differ in several relevant features, such as the estimated rate of recombination and frequency and range of AMR determinants. Although the study lacks detailed epidemiological information, lineage A strains are usually derived from high-risk groups whereas those of lineage $\mathrm{B}$ are more frequently found in heterosexual individuals. The authors speculate about the possibility of lineage A strains becoming adapted to a particular human subpopulation with higher rates of exposure and more chances of coinfection that would facilitate recombination among $N$. gonorrhoeae strains and also with other commensal Neisseria. In addition, these groups are more likely to be treated with antibiotics, not only for $N$. gonorrhoeae infections but also for other STIs. This common practice may have side effects, because the selective pressure exerted by any antibiotic may affect not only the desired target but also to any other species present in the same environment. Even subclinical coinfections of a nontarget species may drive the spread of resistant strains. In fact, this is the likely explanation for the observed prevalence of resistance to sulfonamides and tetracyclines in $N$. gonorrhoeae, again with a higher frequency in lineage A than in lineage B. In this work it is shown that, despite the focus on AMR by both genomic researchers and the media, there is also a subpopulation of N. gonorrhoeae 
susceptible to antimicrobials (lineage B) present in low-risk human groups (mainly heterosexuals and women with asymptomatic infections), and persisting successfully due to the lower probability of exposure to antibiotics [6].

Recent work from Williamson et al. [11] studies 2186 genomes of $N$. gonorrhoeae isolates from Australia. Although it is not a global scale study like the ones mentioned above, it has the advantage of having very detailed information at the patient level. This data allows identification of the relationship between the isolates and the patients' sexual behavior. The authors define more detailed sexual networks, distinguishing within the group of men who have sex with men (MSM), between homosexuals (MSMO) and bisexuals (MSMW), and MSM HIV-positive and MSM HIV-negative pre-exposure prophylaxis users. Their categories also include heterosexual men and women, sex workers and returning international travelers. The combination of these and genomic data allows the authors to identify factors that promote the spread of $N$. gonorrhoeae, such as the use of pre-exposure prophylaxis, which increases the transmission of STIs due to unprotected sex; oropharyngeal gonorrhea in sex workers, disseminating $N$. gonorrhoeae in heterosexual networks; the returning international travelers, more likely to import AMR isolates; and MSMW, which facilitate the connection between MSMO and heterosexual networks. Williamson et al. connect their findings to those of other international studies to discuss the intercontinental emergence of an azithromycinresistant lineage, which they use as a case to emphasize the need for implementing genomic surveillance for the detection of such strains at a global scale.

\section{Conclusion}

These papers illustrate the great power to answer scientific questions afforded by the analysis of complete genome sequences. Here, we would like to underscore that the same information can be used at many different levels, from the long-term evolution of a pathogen to the within individual variation arising in a chronically infected patient by the same pathogen. The progressive adoption of WGS as a regular, even routine, technique in clinical microbiology laboratories and the deposit of those sequences along with basic information in public databases will greatly facilitate epidemiological surveillance. Additional efforts to standardize laboratory and analytical procedures are also necessary to allow interlaboratory and international exploitation of genomic information. However, without complementary information, which cannot be shared openly but should be made available under appropriate circumstances and subjected to reasonable conditions, the full exploitation of genome data will be far from possible.

\section{Author contributions}

All the authors have made a substantial contribution to the work and approved it for publication.

\section{Financial \& competing interests disclosure}

This work was funded by projects BFU2017-89594-R from MICINN (Spanish government) and Prometeo2016-122 (Generalitat Valenciana). C Francés-Cuesta benefits from an FPI predoctoral contract (BES-2015- 074204) from MICINN (Spanish government). The authors have no other relevant affiliations or financial involvement with any organization or entity with a financial interest in or financial conflict with the subject matter or materials discussed in the manuscript apart from those disclosed.

No writing assistance was utilized in the production of this manuscript.

\section{References}

1. Brown NM, Blane B, Raven KE et al. Pilot evaluation of a fully automated bioinformatics system for the analysis of MRSA genomes and detection of outbreaks. J. Clin. Microbiol. 57(11), e00858-19 (2019).

2. Egli A, Saalfrank C, Goldman N et al. Identification of influenza urban transmission patterns by geographical, epidemiological and whole genome sequencing data: protocol for an observational study. BMJ Open 9(8), e030913 (2019).

3. Poon K-S, Tang JW-T, Koay ES-C. HCV genotyping with concurrent profiling of resistance-associated variants by NGS analysis. In: Bioinformatics Tools for Detection and Clinical Interpretation of Genomic Variations. Samadikuchaksaraei A, Seifi M (Eds). IntechOpen, London, UK, 197-209 (2019).

4. Hamers RL, Paredes R. Next-generation sequencing and HIV drug resistance surveillance. Lancet HIV 3(12), e553-e554 (2016).

5. David S, Reuter S, Harris SR et al. Epidemic of carbapenem-resistant Klebsiella pneumoniae in Europe is driven by nosocomial spread. Nat. Microbiol. 4(11), 1919-1929 (2019).

6. Sánchez-Busó L, Golparian D, Corander J et al. The impact of antimicrobials on gonococcal evolution. Nat. Microbiol. 4(11), 1941-1950 (2019).

7. Nakayama S-I, Shimuta K, Furubayashi K-I, Kawahata T, Unemo M, Ohnishi M. New ceftriaxone- and multidrug-resistant Neisseria gonorrhoeae strain with a novel mosaic penA gene isolated in Japan. Antimicrob. Agents Chemother. 60(7), 4339-4341 (2016). 
8. Tapsall JW, Ndowa F, Lewis DA, Unemo M. Meeting the public health challenge of multidrug- and extensively drug-resistant Neisseria gonorrhoeae. Expert Rev. Anti. Infect. Ther. 7(7), 821-834 (2009).

9. Bush K, Courvalin P, Dantas G et al. Tackling antibiotic resistance. Nat. Rev. Microbiol. 9(12), 894-896 (2011).

10. Arora N, Schuenemann VJ, Jäger G et al. Origin of modern syphilis and emergence of a pandemic Treponema pallidum cluster. Nat. Microbiol. 2, 16245 (2016).

11. Williamson DA, Chow EPF, Gorrie CL et al. Bridging of Neisseria gonorrhoeae lineages across sexual networks in the HIV pre-exposure prophylaxis era. Nat. Commun. 10(1), 3988 (2019). 